\title{
Resonant X-ray Raman scattering for Al, Si and their oxides
}

\author{
J. Szlachetko ${ }^{\text {a,* }}$, M. Berset a, J.-Cl. Dousse a , K. Fennane a, M. Szlachetko ${ }^{\text {a }}$, \\ R. Barrett ${ }^{\text {b }}$, J. Hoszowska ${ }^{\text {b }}$, A. Kubala-Kukus ${ }^{\text {c }}$, M. Pajek ${ }^{\text {c }}$ \\ a Department of Physics, University of Fribourg, CH-1700 Fribourg, Switzerland \\ b European Synchrotron Radiation Facility (ESRF), F-38043 Grenoble, France \\ c Swietokrzyska Academy, Institute of Physics, 25-406 Kielce, Poland
}

Available online 1 August 2005

\begin{abstract}
High-resolution measurements of the resonant X-ray Raman scattering (RRS) of Al and Si and their oxides were performed at the European Synchrotron Radiation Facility (ESRF) in Grenoble, France, using a von Hamos Bragg-type curved crystal spectrometer. To probe the influence of chemical effects on the RRS X-ray spectra, $\mathrm{Al}_{2} \mathrm{O}_{3}$ and $\mathrm{SiO}_{2}$ samples were also investigated. The X-ray RRS spectra were measured at different photon beam energies tuned below the K-absorption edge. The measured spectra are compared to results of RRS calculations based on the second-order perturbation theory within the Kramers-Heisenberg approach.
\end{abstract}

PACS: $32.30 . \mathrm{Rj} ; 32.70 . \mathrm{Jz} ; 32.80 . \mathrm{Ys} ; 31.15 . \mathrm{Md}$

Keywords: X-ray RRS; TXRF; High-resolution XES; Second order perturbation theory

\section{Introduction}

The application of the total reflection X-ray fluorescence (TXRF) method [1] combined with intense synchrotron X-ray sources offers new possibilities for measuring very low concentrations of light element impurities on the surface of Si wa-

\footnotetext{
* Corresponding author. Tel.: +41 26300 9080; fax: +41 26 3009747.

E-mail address: jakub.szlachetko@unifr.ch (J. Szlachetko).
}

fers. Several experiments $[2,3]$ have shown that the detection limit of $\mathrm{Al}$ impurities on Si surface is limited by the presence of the X-ray resonant Raman scattering (RRS). For photon excitation energies tuned below the Si K-absorption edge to avoid the intense $\mathrm{Si} \mathrm{K}$ fluorescence X-ray line, the RRS structure of Si is indeed overlapping with the Al K X-ray peak. In the analysis of low-resolution TXRF data a precise knowledge of the background profile that is mainly due to the X-ray Raman scattering is therefore crucial. By now, only theoretical predictions [4] were available 
to determine the shape of the RRS structure. However, the assumptions made in these calculations and the resulting uncertainties on the theoretical RRS shape diminish substantially the sensitivity of the TXRF method.

\section{Experiment}

The RRS X-ray spectra of metallic Al, polycrystalline $\mathrm{Si}$ and amorphous $\mathrm{Al}_{2} \mathrm{O}_{3}$ and $\mathrm{SiO}_{2}$ samples were measured at the ESRF beamline ID21 by means of high-resolution X-ray spectroscopy, using a von Hamos Bragg-type curved crystal spectrometer [5]. As the Bragg angle domain covered by this instrument extends from $24.4^{\circ}$ to $61.1^{\circ}$, for the observation of the 1400-1800 eV X-ray spectra of interest the spectrometer was equipped with an Ammonium Dihydrogen Phosphate ADP (1 01 ) crystal $(2 d=10.642 \AA)$. The diffracted X-rays were recorded with a $26.8 \mathrm{~mm}$ long and $8 \mathrm{~mm}$ high position-sensitive back illuminated CCD (Charge Coupled Device) detector consisting of 1340 columns and 400 rows with a pixel size of $20 \times 20 \mu \mathrm{m}^{2}$. At ID21 the spectrometer was installed downstream of the Scanning Transmission X-ray Microscope (STXM) chamber to which it was connected through a $\sim 200 \mathrm{~cm}$ long evacuated pipe.

The X-ray beam was monochromatized by means of two $20 \AA \mathrm{Ni} / \mathrm{B} 4 \mathrm{C}$ multilayers. Residual higher energy photons were suppressed with a $\mathrm{Ni}$ mirror. Thanks to this monochromator $10^{12}-10^{13}$ incident photons/s were obtained on the targets with an energy resolution of $\sim 6 \mathrm{eV}$. For all measurements the angle between the incoming beam and the target surface was $20^{\circ} .25 \mathrm{~mm}$ high $\times 12 \mathrm{~mm}$ wide $\times 1 \mathrm{~mm}$ thick solid targets were used. The specified purity of the targets was better than $99.99 \%$ for $\mathrm{Al}$ and $\mathrm{Si}$ and $97.5 \%$ and $99.9 \%$, respectively, for $\mathrm{Al}_{2} \mathrm{O}_{3}$ and $\mathrm{SiO}_{2}$.

Data were taken at several excitation energies between $1790 \mathrm{eV}$ and $1900 \mathrm{eV}$ for $\mathrm{Si}$ and $\mathrm{SiO}_{2}$, respectively between $1540 \mathrm{eV}$ and $1600 \mathrm{eV}$ for $\mathrm{Al}$ and $\mathrm{Al}_{2} \mathrm{O}_{3}$. For illustration, the $\mathrm{Si}$ and $\mathrm{SiO}_{2}$, respectively $\mathrm{Al}$ and $\mathrm{Al}_{2} \mathrm{O}_{3}$, RRS spectra are shown in Figs. 1 and 2 for three different excitation energies. The beam energy calibration was determined from measurements of the K-absorption edges of

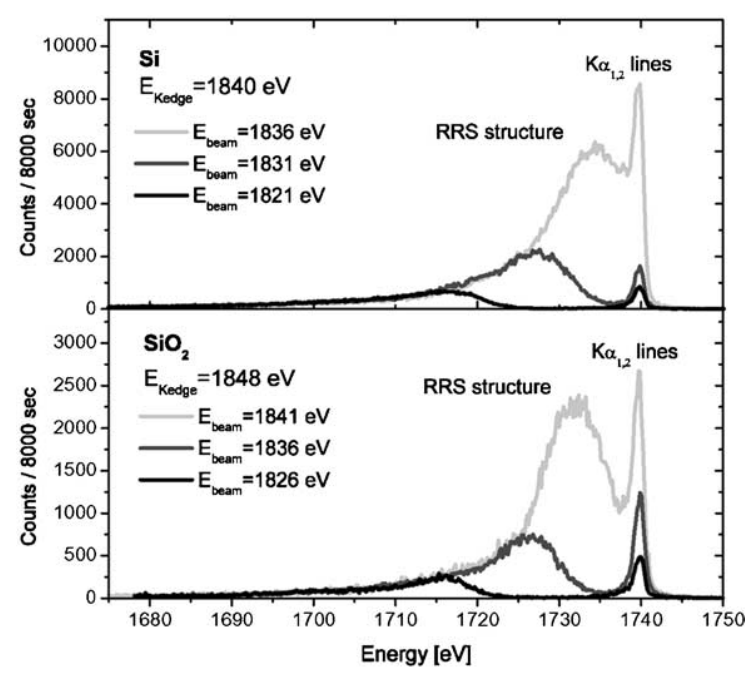

Fig. 1. High-resolution RRS spectra of $\mathrm{Si}$ and $\mathrm{SiO}_{2}$ measured for different excitation energies.

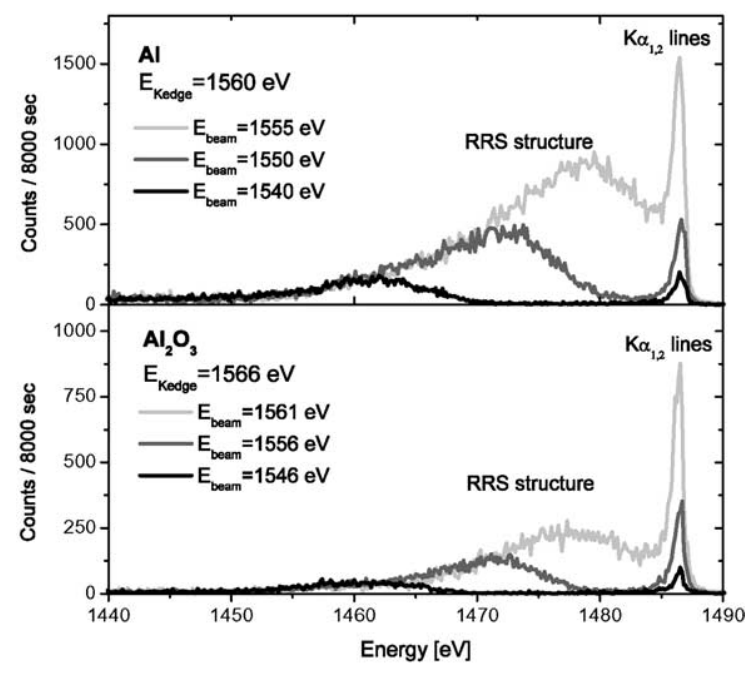

Fig. 2. High-resolution RRS spectra of $\mathrm{Al}$ and $\mathrm{Al}_{2} \mathrm{O}_{3}$ measured for different excitation energies.

$\mathrm{Si}, \mathrm{SiO}_{2}, \mathrm{Al}$ and $\mathrm{Al}_{2} \mathrm{O}_{3}$. For each target the intensities of the different spectra were normalized off-line for the number of incident photons and acquisition time and corrected for the beam intensity profile (in the von Hamos slit-geometry, different regions of the crystal surface view different parts of the target so that the shape of the measured spectrum is slightly affected by the spatial 
distribution of the beam intensity). The energy calibration of the crystal spectrometer was deduced from measurements of the $\mathrm{K} \alpha_{1,2} \mathrm{X}$-ray lines of $\mathrm{Al}$ and $\mathrm{Si}$. The same measurements were employed to determine the instrumental broadening of the spectrometer.

\section{Results and interpretation}

In order to better understand the structures of the measured RRS X-ray spectra, calculations of the resonant Raman scattering around the $\mathrm{K}$-absorption edge with a $2 \mathrm{p}$ vacancy in the final state were performed, using the second-order perturbation theory based on the Kramers-Heisenberg formula [6-8]. A more detailed description of these calculations can be found in [9]. An example of a theoretical RRS shape calculated within this model is shown in Fig. 3(a). For comparison with experimental data the theoretical RRS spectra were convoluted with the Gaussian instrumental response of the spectrometer $(\sigma \cong 0.5 \mathrm{eV})$ and corrected for the beam energy profile, assuming for the latter a Gaussian shape with a full width at half maximum of $\sim 6 \mathrm{eV}$.

As an example, the Si RRS spectrum observed at an excitation energy of $1821 \mathrm{eV}$ is compared with the corresponding convoluted theoretical spectrum (thick solid line) in Fig. 3(b). The two thin solid lines whose maxima lie around $1718 \mathrm{eV}$ correspond to the RRS profiles computed for a $\mathrm{L}_{2}$ or $\mathrm{L}_{3}$ vacancy in the final state. As shown, the theoretical RRS shape is in good agreement with the experimental data. The $\mathrm{K} \alpha_{1,2}$ fluorescence lines which are also visible in Fig. 3(b) are mostly due to the high-energy tail of the beam energy distribution. The two lines were fitted each with a single Voigt profile. Calculations performed for a perfectly monochromatic beam have shown that the centroid positions, shapes and linewidths of the $\mathrm{K} \alpha_{1,2}$ transitions are slightly varying when the beam energy is tuned by $\pm 5 \mathrm{eV}$ around the K-edge [9]. The small differences observed in Fig. 3(b) between the measured and fitted $\mathrm{K} \alpha_{1,2}$ lines are probably due to this effect as a consequence of the finite beam energy resolution. The intensity excess found in the
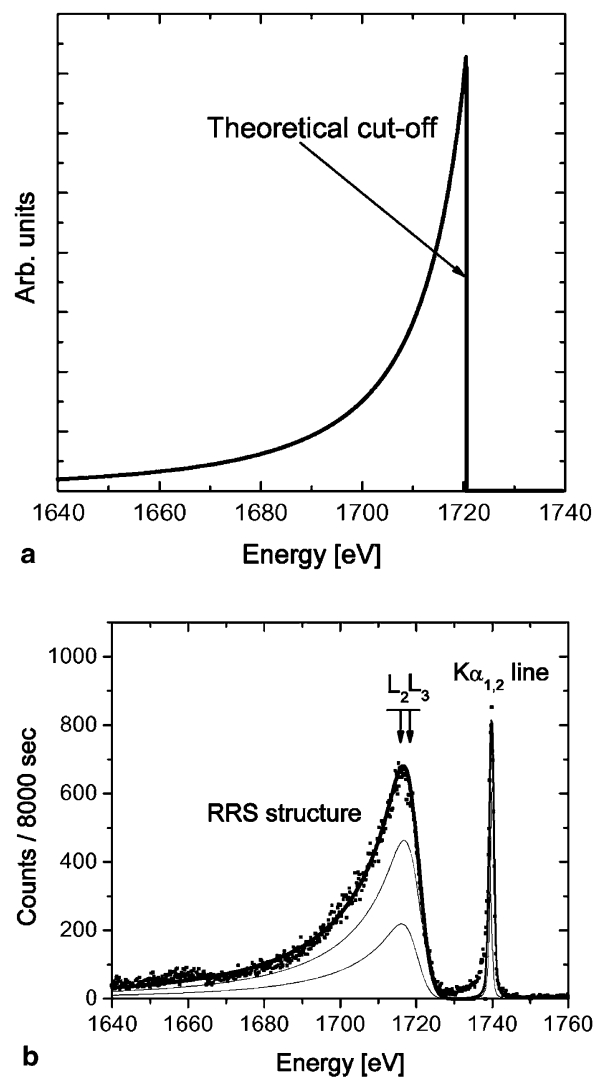

Fig. 3. (a) Theoretical shape of the RRS structure of Si for a beam energy of $1821 \mathrm{eV}$. The cut-off energy is given by: $\omega_{\text {cut-off }}=\omega_{\text {beam }}-\omega_{\mathrm{L}}$, where $\omega_{\mathrm{L}}$ stands for the average binding energy of the $\mathrm{L}_{2,3}$ subshells. (b) High-resolution RRS spectrum of $\mathrm{Si}$ for an excitation energy of $1821 \mathrm{eV}$. The dots correspond to the experimental data, the thick solid line to the theoretical shape obtained from the sum of the two RRS profiles (thin solid lines) discussed in the text.

low-energy tails of the $\mathrm{K} \alpha_{1,2}$ lines corresponds to the excitation of $\mathrm{K}$-shell electrons into the M-shell and quasi-simultaneous filling of the 1 s holes by $\mathrm{L}_{2,3}$ electrons.

An improved analysis of the measured RRS spectra, that needs further calculations to describe the details of the observed features and to determine the influence of the chemical effects on the RRS spectra, will be performed. On the other hand, the measured RRS spectra of Si will be compared to the calculations of Gavrila [4], because the latter have been extensively used to determine the RRS background in low-resolution TXRF spectra. 


\section{Acknowledgements}

The authors would like to thank Dr. R. Tucoulou and his collaborators of the beam-line ID21 for their excellent support during the experiment. This work was supported by the Swiss National Science Foundation and by the ESRF.

\section{References}

[1] R. Klockenkämper, Total Reflection X-ray Fluorescence Analysis, Wiley, New York, 1997.
[2] K. Baur, J. Kerner, S. Brennan, A. Singh, P. Pianetta, J. Appl. Phys. 88 (2000) 4624.

[3] K. Baur, S. Brennan, B. Burrow, D. Werho, P. Pianetta, Spectrochem. Acta B 56 (2001) 2049.

[4] M. Gavrila, Rev. Roum. Phys. 19 (1974) 473.

[5] J. Hoszowska, J.-Cl. Dousse, J. Kern, Ch. Rhême, Nucl. Instr. Meth. Phys. Res. A 376 (1996) 129.

[6] T. Åberg, in: B. Crasemann (Ed.), Atomic Inner-Shell Physics, Plenum Press, New York, 1985, p. 419.

[7] F. Gel'mukhanov, H. Agren, Phys. Rep. 312 (1999) 87.

[8] A. Kotani, S. Shin, Rev. Mod. Phys. 73 (2001) 203.

[9] J. Tulkki, T. Åberg, J. Phys. B 15 (1982) L435. 